University of Nebraska - Lincoln

DigitalCommons@University of Nebraska - Lincoln

April 1974

\title{
Angular Distribution of Photoelectrons from Atomic Oxygen, Nitrogen, and Carbon
}

\author{
Steven T. Manson \\ Georgia State University Atlanta, Georgia \\ David J. Kennedy \\ Georgia Institute of Technology, Atlanta, Georgia \\ Anthony F. Starace \\ University of Nebraska-Lincoln, astarace1@unl.edu \\ Dan Dill \\ Boston University, Boston, Massachusetts
}

Follow this and additional works at: https://digitalcommons.unl.edu/physicsstarace

Part of the Physics Commons

Manson, Steven T.; Kennedy, David J.; Starace, Anthony F.; and Dill, Dan, "Angular Distribution of Photoelectrons from Atomic Oxygen, Nitrogen, and Carbon" (1974). Anthony F. Starace Publications. 149. https://digitalcommons.unl.edu/physicsstarace/149

This Article is brought to you for free and open access by the Research Papers in Physics and Astronomy at DigitalCommons@University of Nebraska - Lincoln. It has been accepted for inclusion in Anthony F. Starace Publications by an authorized administrator of DigitalCommons@University of Nebraska - Lincoln. 


\title{
Angular Distribution of Photoelectrons from Atomic Oxygen, Nitrogen, and Carbon
}

\author{
Steven T. Manson \\ Department of Physics, Georgia State University Atlanta, Georgia 30303, U.S.A. \\ David J. Kennedy \\ School of Physics, Georgia Institute of Technology, Atlanta, Georgia 30332, U.S.A. \\ (Present address: School of Physical Sciences, New University of Ulster, Coleraine, Northern Ireland) \\ Anthony F. Starace \\ Behlen Laboratory of Physics, University of Nebraska-Lincoln, Lincoln, Nebraska 68508, U.S.A. \\ Dan Dill \\ Department of Chemistry, Boston University, Boston, Massachusetts 02215, U.S.A.
}

\begin{abstract}
The angular distributions of photoelectrons from atomic oxygen, nitrogen and carbon are calculated. Both Hartree-Fock and Hartree-Slater (Herman-Skillman) wave functions are used for oxygen, and the agreement is excellent; thus only Hartree-Slater functions are used for carbon and nitrogen. The pitch angle distribution of photoelectrons is discussed and it is shown that previous approximations of energy independent isotropic or $\sin ^{2} \theta$ distributions are at odds with our results, which vary with energy. This variation with energy is discussed as is the reliability of these calculations.
\end{abstract}

\section{Introduction}

The process of photoionization plays a significant role in many aeronomic and astrophysical phenomena (Dalgarno, 1967; Takayanagi and Itikawa, 1970). If the process occurs in a dense medium, the only important quantity is the photoabsorption cross section; the angular distribution of the photoelectrons is inconsequential, since it rapidly becomes isotropic due to collisions with the atoms and molecules of the medium. In a less dense medium, where the photoelectrons can travel considerable distances before losing their energy, the initial photoelectron angular distribution can be important. An example of such a situation occurs in the upper $F 2$ layer of the Earth's upper atmosphere where photoelectrons produced by solar radiation ionizing atomic oxygen (produced by solar photodissociation of $\mathrm{O}_{2}$ ) can either deposit their energy locally or spiral along the lines of the Earth's magnetic field and deposit their energy nonlocally at the conjugate point (Mariani, 1964; Whitten, 1968; Fontheim, 1968; Kwei and Nesbit, 1968), i.e. magnetic focusing of the photoelectrons by the Earth's magnetic field.

In this paper, the angular distribution of photoelectrons from atomic oxygen is investigated using Hartree-Fock (HF) wave functions. A previous work (Kennedy and Manson, 1972a) used Hartree-Slater (HS) wave functions in an incorrect theoretical formulation. Here the correct formulation is used to compare HS and HF results. Agreement between these results is so good that we have extended the HS calculations to atomic nitrogen and carbon as well. 
In Section II a brief discussion of the theory of photoionization and photoelectron angular distributions and of the method of calculation is given. A derivation of the formulae relevant to the conjugate point phenomenon is presented in Section III. In Section IV, we present and discuss the results of our calculations, while Section V contains some final remarks and recapitulates the major results of this paper.

\section{Theory of Photoionization and Photoelectron Angular Distributions}

If we assume the wave functions of an atomic system can be well approximated by antisymmetrized products of single particle wave functions (labeled $P_{n l}{ }^{i}$ and $P_{n l} f$ for initial and final states respectively), then the general expression for photoionization of an electron from an $(n l)^{p 2 S+1} L$ state to an $\left\{\left[(n l)^{q-12 S_{c}+1} L_{c}\right],\left(\varepsilon l^{\prime}\right)\right\}^{2 S+1} L^{\prime}$ state is given by (Bates, 1946)

$$
\sigma_{n l}\left(L S, L_{c} S_{c}, \varepsilon l^{\prime} L^{\prime}\right)=\frac{4 \pi^{2} \alpha{a_{0}}^{2}}{3 \omega_{i}} \frac{I+\varepsilon}{R} \frac{1}{4 l_{>}^{2}-1} \zeta\left(L S, L_{c} S_{c}, l^{\prime} L^{\prime}\right) \gamma\left|R_{l^{\prime}}(\varepsilon)\right|^{2}
$$

Here $I$ is the experimental ionization potential of the ${ }^{2 S+1} L$ state of the atom relative to the ${ }^{2 S_{c}+1} L_{c}$ state of the residual ion core, $\varepsilon$ is the photoelectron energy, $R$ the Rydberg energy, $\alpha$ the fine structure constant, $a_{0}$ the Bohr radius, $\omega_{i}$ the statistical weight of the initial state, $l^{\prime}$ the final photoelectron angular momentum, $l_{>}$is the greater of $l$ and $l^{\prime}$, and $\zeta$, the relative multiplet strength. The overlap integral $\gamma$ and the radial dipole matrix element $R_{l^{\prime}}(\varepsilon)$ are given by

$$
\gamma=\prod_{\substack{\text { passive } \\ \text { electrons }}}\left|\int_{0}^{\infty} P_{n l}^{i}(r) P_{n l}^{f}(r) \mathrm{d} r\right|^{2}
$$

and

$$
R_{l^{\prime}}(\varepsilon)=\int_{0}^{\infty} P_{n l}^{i}(r) r P_{\varepsilon l^{\prime}}^{f}(r) \mathrm{d} r
$$

where the continuum wave function is normalized such that

$$
P_{\varepsilon l^{\prime}}^{f}(r)_{r \rightarrow \infty} \rightarrow \pi^{-1 / 2} \varepsilon^{-1 / 4} \sin \left(\varepsilon^{1 / 2} r+\varepsilon^{-1 / 2} \ln 2 \varepsilon^{1 / 2} r-\frac{1}{2} l \pi+\sigma_{l^{\prime}}+\delta_{l^{\prime}}\right)
$$

where $\sigma_{l^{\prime}}=\arg \Gamma\left(l^{\prime}+1-i \varepsilon^{-1 / 2}\right)$ is the Coulomb phase shift and where $\delta_{l^{\prime}}$ is the phase shift with respect to Coulomb waves. This normalization of $P_{\varepsilon l^{\prime}}^{f}$ is the usual normalization of continuum wave functions per unit energy range.

The single particle wave functions for the initial discrete states were chosen to be Hartree-Fock HF functions compiled by Clementi (1965); the single particle discrete functions for the positive ion cores were similarly chosen to be the HF functions of Clementi (1965). The wave function for the final continuum electron is obtained in the field of the HF positive ion core. The numerical details are given by Manson and Cooper (1968) and Kennedy and Manson (1972b), and the continuum HF equations have been presented in detail by Dalgarno, Henry and Stewart (1964).

In addition Herman-Skillman (HS) wave functions have also been employed in these calculations for comparison with the HF results. In using these wave functions, no core relaxation is considered, and the continuum function is found from the central potential appropriate to the ground state of the atom. A full discussion of this type of calculation is given by Manson and Cooper (1968). 
The angular distribution of photoelectrons from an initial ${ }^{2 S+1} L$ state to a ${ }^{2 S_{c}+1} L_{c}$ state of the residual ion core is given for unpolarized light by (Cooper and Zare, 1968, 1969).

$$
\frac{\mathrm{d} \sigma_{i}(\varepsilon)}{\mathrm{d} \Omega}=\frac{\sigma_{i}(\varepsilon)}{4 \pi}\left[1-\frac{\beta_{i}(\varepsilon)}{2} P_{2}(\cos \theta)\right]
$$

where

$$
\sigma_{i}(\varepsilon)=\sum_{L^{\prime}} \sum_{l^{\prime}=l-1}^{l+1} \sigma_{n l}\left(L S, L_{c} S_{c}, \varepsilon l^{\prime} L^{\prime}\right)
$$

$\theta$ is the angle between the incident photon beam and photoelectron direction, $P_{2}(x)=1 / 2\left(3 x^{2}-1\right)$ and $\beta_{i}(\varepsilon)$ is the asymmetry parameter. The form of the angular distribution (Equation 5) can be obtained from quite general considerations depending only on the absorption of radiation by an unpolarized target occurring via an electric dipole process (Yang, 1948); deviation from the form of this equation implies the presence of absorption via processes other than electric dipole. The asymmetry parameter, $\beta_{i}(\varepsilon)$, however, does depend upon the details of the calculation. Specifically, for the $L S$ coupled anti-symmetric products of single particle Hartree-Fock functions we are using, the asymmetry parameter is a sum over the $\beta$ 's corresponding to each of the possible values of the angular momentum $j_{t}$ transferred to the atom by the photon, each of these $\beta$ 's being weighted by the relative cross section for each value of $j_{t}$ (Dill and Fano, 1972; Fano and Dill, 1972; Dill, 1973)

$$
\beta_{i}=\sum_{j t} \beta\left(j_{t}\right) \sigma\left(j_{t}\right) / \sum_{j t} \sigma\left(j_{t}\right)
$$

Transitions where the parity change of the target is $(-1)^{j_{t}}$ are said to be parity favored and $\beta\left(j_{t}\right)$ is given by (Dill and Fano, 1972; Dill, 1973)

$$
\begin{aligned}
& \beta\left(j_{t}\right)_{\mathrm{fav}} \\
& =\frac{\left(j_{t}+2\right)\left|S_{+}\left(j_{t}\right)\right|^{2}+\left(j_{t}-1\right)\left|S_{-}\left(j_{t}\right)\right|^{2}-3\left[j_{t}\left(j_{t}+1\right)\right]^{1 / 2}\left[S_{+}\left(j_{t}\right) S_{-} *\left(j_{t}\right)+S_{+}{ }^{*}\left(j_{t}\right) S_{-}\left(j_{t}\right)\right]}{\left(2 j_{t}+1\right)\left[\left|S_{+}\left(j_{t}\right)\right|^{2}+\left|S_{-}\left(j_{t}\right)\right|^{2}\right]}
\end{aligned}
$$

where $\bar{S}_{ \pm}\left(j_{t}\right)$ denote the photoionization amplitude for a given $j_{t}$ and for $l^{\prime}=j_{t} \pm 1$; values of $j_{t}$ for which the parity change is $-(-1)^{j_{t}}$ are called parity unfavored and have a $\sin ^{2} \theta$ distribution (Dill and Fano, 1972; Dill, 1973), i.e.

$$
\beta\left(j_{t}\right)_{\mathrm{unf}}=-1,
$$

independently of dynamics. The integrated cross sections $\sigma\left(j_{t}\right)$ have the structure (Dill, 1973)

$$
\begin{aligned}
& \sigma\left(j_{t}\right)_{\mathrm{fav}}=\pi \lambda^{2} \frac{2 j_{t}+1}{2 J_{0}+1}\left[\left|S_{+}\left(j_{t}\right)\right|^{2}+\left|S_{-}\left(j_{t}\right)\right|^{2}\right] \\
& \sigma\left(j_{t}\right)_{\mathrm{unf}}=\pi \lambda^{2} \frac{2 j_{t}+1}{2 J_{0}+1}\left|S_{0}\left(j_{t}\right)\right|^{2}
\end{aligned}
$$

where $\bar{S}_{0}\left(j_{t}\right)$ is the photoionization amplitude for the value of $j_{t}=l^{\prime}$ and is $\lambda$ the photon wavelength (divided by $2 \pi$ ). Detailed discussion of these matrix elements is given by Dill, Manson, and Starace (1974). As an example of the results, $\beta_{i}$ for the ${ }^{3} P \rightarrow{ }^{2} D$ photoionization (labeled by $i$ ) for oxygen is given by

$$
\beta=\frac{3\left|S_{d, i}^{\prime}(1)\right|^{2}-3 \sqrt{2}\left[S_{d, i}^{\prime}(1) S_{s, i}^{\prime}(1)^{*}+c c\right]-5\left[\left|S_{d, i}^{\prime}(2)\right|^{2}-\left|S_{d, i}^{\prime}(3)\right|^{2}\right]}{3\left[\left|S_{s, i}^{\prime}(1)\right|^{2}+\left|S_{d, i}^{\prime}(1)\right|^{2}\right]+5\left|S_{d, i}^{\prime}(2)\right|^{2}+7\left|S_{d, i}^{\prime}(3)\right|^{2}},
$$


where $c c$ is a complex congugate and

$$
\begin{aligned}
& \bar{S}_{s, i}^{\prime}(1)=\frac{1}{3} \mathrm{e}^{i\left(\sigma_{s}+\delta_{s, i}{ }^{3} D\right)} R_{s, i}^{3} \\
& S_{d, i}^{\prime}(1)=\frac{\sqrt{2}}{5} \mathrm{e}^{i \sigma_{d}}\left[\frac{1}{3} \mathrm{e}^{i \delta_{d, i}{ }^{3}{ }^{S}} R_{d, i}^{3 S}+\frac{3}{4} \mathrm{e}^{i \delta_{d, i}{ }^{3}{ }^{3}} R_{d, i}^{3 P}+\frac{7}{12} \mathrm{e}^{i \delta_{d, i}{ }^{3} D} R_{d, i}^{3 D}\right] \\
& \bar{S}_{d, i}^{\prime}(1)=\frac{\sqrt{2}}{5} \mathrm{e}^{i \sigma_{d}}\left[\frac{-1}{3} \mathrm{e}^{i \delta_{d, i}{ }^{3} S} R_{d, i}^{3_{S}}+\frac{-1}{4} \mathrm{e}^{i \delta_{d, i}{ }^{3} P} R_{d, i}^{3 P}+\frac{7}{12} \mathrm{e}^{i \delta_{d, i}{ }^{3} D} R_{d, i}^{3_{D}}\right] \\
& S_{d, i}^{\prime}(1)=\frac{\sqrt{2}}{5} \mathrm{e}^{i \sigma_{d}}\left[\frac{1}{3} \mathrm{e}^{i \delta_{d, i}{ }^{3} S} R_{d, i}^{3_{S}}+\frac{-1}{2} \mathrm{e}^{i \delta_{d, i}{ }^{{ }^{3} P}} R_{d, i}^{3^{3} P}+\frac{1}{6} \mathrm{e}^{i \delta_{d, i}{ }^{3} D} R_{d, i}^{3_{D}}\right]
\end{aligned}
$$

in which $\sigma_{s}$ and $\sigma_{d}$ are the Coulomb phase shifts defined previously. The superscripts on the phase shifts and dipole matrix elements refer to the different total angular momentum states of the photoelectron-ion system after photoionization: e.g. a $d$-wave photoelectron plus a ${ }^{2} D$ ion core can couple to possible final states ${ }^{3} S,{ }^{3} P$, and ${ }^{3} D$.

Note that the second term in the numerator of Equation (13) introduces a cosine of the difference of the phase shifts between the $d$ - and $s$-continua very much like the simple Cooper-Zare expression (Cooper and Zare, 1968, 1969). The other terms, which do not appear in the Cooper-Zare formulation, introduce the cosine of phase shift differences between the various possible $d$-continua $\left({ }^{3} S\right.$, ${ }^{3} P$, or $\left.{ }^{3} D\right)$. However, it is seen from Equations (12b-d) that if the phase shifts and dipole matrix elements of $d$-continua are the same, (as they are in a HS type calculation), the $j_{t}=2$ and $j_{t}=3$ contributions vanish and Equation (11) reduces to the Cooper-Zare formula for an initial $p$-state,

$$
\beta_{i}=2 \frac{R_{d}{ }^{2}-2 R_{s} R_{d} \cos \left(\xi_{d}-\xi_{s}\right)}{2 R_{d}{ }^{2}+R_{s}{ }^{2}}
$$

where $\xi_{l \pm 1}=\delta_{l \pm 1}+\sigma_{l \pm 1}$. Equation (15) shows that within the HS approximation, $\beta_{i}(\varepsilon)$ is the same for each channel and depends only upon the photoelectron energy, $\varepsilon$. Thus when a HF calculation results in differing matrix elements and phase shifts for differing final state multiplets with the same ion core, the Cooper-Zare formula will be in error to an extent determined by the magnitude of these phase shift differences. In closing this section we emphasize that Equation (15) is applied to the angular distribution of all photoelectrons in a transition from a given atomic state to a given ionic state. The discussion of this point in a previous paper (Kennedy and Manson, 1972b) is incorrect and should be disregarded. The $\beta$ results in that paper for the ${ }^{2} S \rightarrow{ }^{2} P$ channel is the correct one for all channels in the Cooper-Zare approximation, whose validity for oxygen is examined below.

\section{Pitch Angle Distribution of Photoelectrons}

For the conjugate point phenomenon, we are interested in the angular distribution of photoelectrons with respect to the Earth's magnetic field direction, i.e. the pitch angle distribution. To get this, we have the situation shown in Figure 1 where the magnetic field is in the $z$-direction and the incident photon is in the $x-z$ plane, making an angle $\gamma$ with the $z$-axis. Assume the photoionization takes 


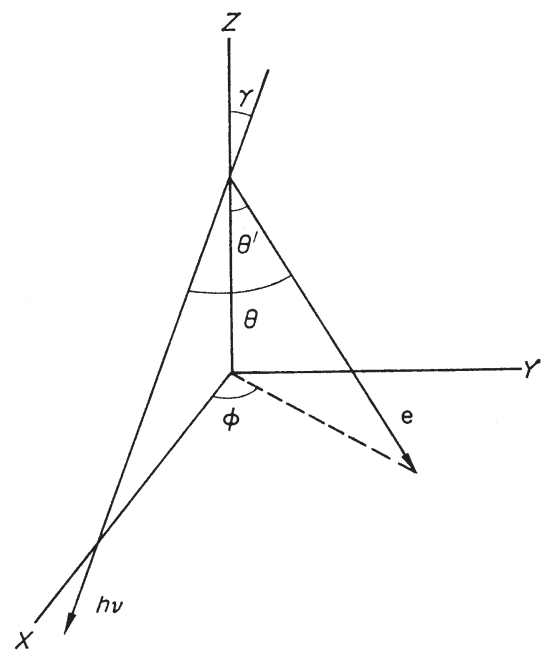

Figure 1. Schematic Representation of the Photoionization Process.

place on the $z$-axis with the photoelectron going in the direction shown. The angular distribution of photoelectrons is given generally by

$$
\frac{\mathrm{d} \sigma}{\mathrm{d} \Omega}=f(\cos \theta)=\sum_{n} A_{n} P_{n}(\cos \theta)=\sum_{n} \frac{4 \pi}{2 n+1} A_{n} \sum_{m=-n} Y_{n}{ }^{m}(\gamma, 0) Y_{n}{ }^{m}\left(\theta^{\prime}, \phi\right)
$$

using the addition theorem for spherical harmonics. We are interested in the pitch angle distribution, $\mathrm{d} \sigma / \mathrm{d}\left(\cos \theta^{\prime}\right)$ without regard to $\phi$. Thus, integrating over $\phi$, we obtain

$$
\frac{\mathrm{d} \sigma}{\mathrm{d}\left(\cos \theta^{\prime}\right)}=2 \pi \sum_{n} A_{n} P_{n}(\cos \gamma) P_{n}\left(\cos \theta^{\prime}\right)
$$

For an electric dipole interaction $A_{0}=\sigma /(4 \pi)$ and $A_{2}=-(\beta / 2) A_{0}$, so that

$$
\frac{\mathrm{d} \sigma}{\mathrm{d}\left(\cos \theta^{\prime}\right)}=\frac{\sigma}{2}\left[1-\frac{\beta}{2} P_{2}(\cos \gamma) P_{2}\left(\cos \theta^{\prime}\right)\right],
$$

which is the pitch angle distribution.

\section{Results and Discussion}

Calculations have been performed for photoionization of the $2 p$ subshell of oxygen from the ground configuration in the ${ }^{3} P,{ }^{1} S$, and ${ }^{1} D$ states in both HF and HS approximations as discussed in Section II. The calculated cross section for photoionization from the ${ }^{3} P$ state of the ground $(2 p)^{4}$ configuration of neutral oxygen is given in Figure 2. The results show fairly good agreement among the HF-length (HFL), HF-velocity (HFV), and HS formulations. In addition, our results agree essentially exactly with the HF results of Dalgarno et al. (1964). This is, of course, to be expected and serves to confirm our computational technique.

More importantly, it has been shown that the angular distribution of photoelectrons is only very weakly a function of the cross section; it is the phase shifts of the continuum waves which are all-important (Manson and Kennedy, 1970; Kennedy and Manson, 1972b). This is true in any spectral re- 


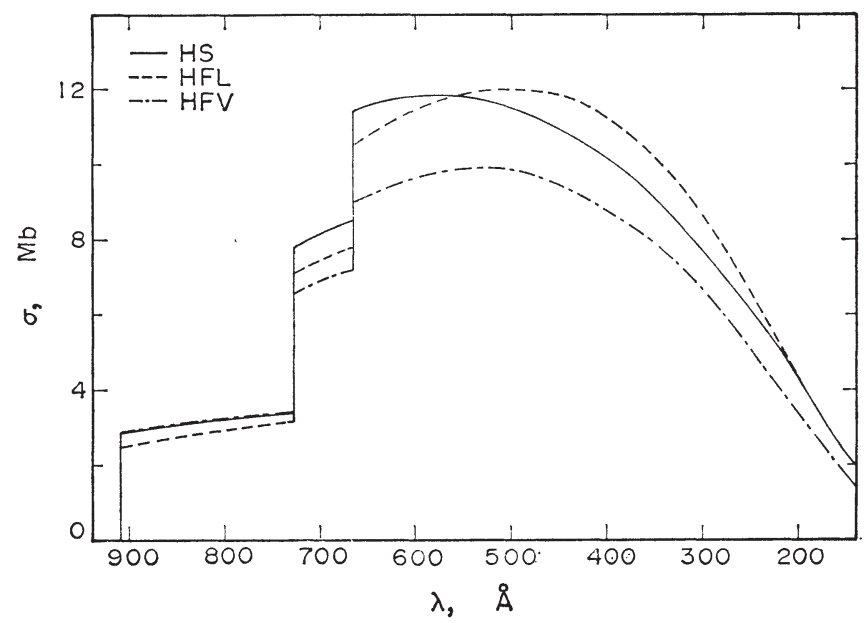

Figure 2. Photoionization cross section of $\mathrm{O}\left({ }^{3} P\right) \rightarrow \mathrm{O}^{+}\left({ }^{4} S,{ }^{2} D,{ }^{2} P\right)+e$. The solid curve is the HartreeSlater (Hs) result, and the dashed and dot-dashed curves are the Hartree-Fock Length (HFL) and Velocity (HFV) respectively.

gion where neither a shape resonance nor a Cooper minimum occurs; we note that neither is in evidence for the photoionization of oxygen. Further, the phase shifts of the various $\varepsilon$ d's in the HF and HS approximation are essentially the same. Thus it is expected that the angular distribution parameters, the $\beta_{i}$ 's, will not differ very much in the various approximations. This is borne out by Figure 3 , which shows the $\beta_{i}$ 's for the various possible photoionization transitions computed in the HFL and HS approximations. The agreement among the several approximations is seen to be excellent. Thus we conclude that for light atoms, the HS approximation will predict excellent $\beta$ 's and the CooperZare formulation is adequate.

In the previous work relating to the conjugate point phenomenon, the angular distribution parameter was assumed to be isotropic $(\beta=0)$ by Fontheim et al. (1968) or $\sin ^{2} \theta(\beta=2)$ by Mariani

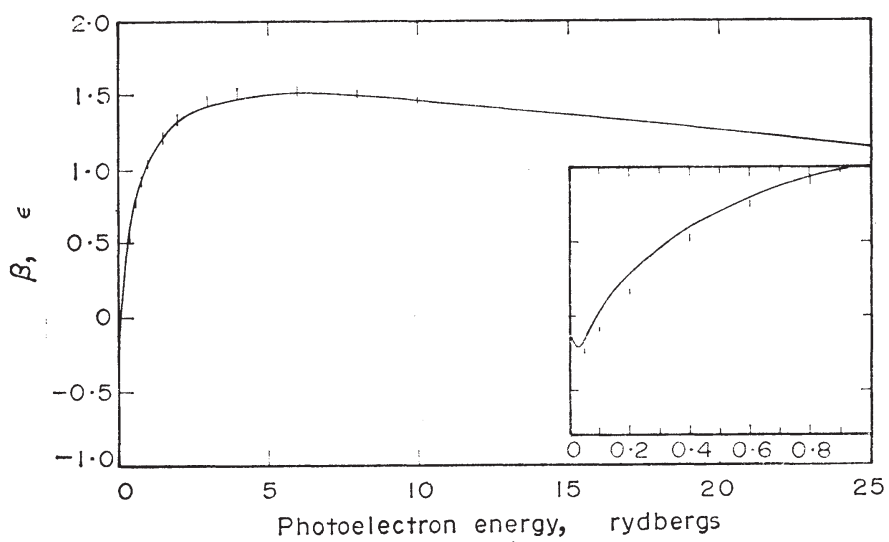

Figure 3. Asymmetry parameter, $\beta(\varepsilon)$, for $2 p$ photoionization of atomic oxygen. The solid curve is the HS result and the vertical lines represent the range of HFL results for all the photoionization channels from the $(2 p)^{4}$ atomic configuration to $(2 p)^{3}$ ionic configuration. 
(1964) and Whitten (1968). From Figure 3, however, it is apparent that the $\beta$ 's vary with energy. Further, an isotropic distribution is valid only just above threshold, and $\beta_{i}$ never gets near 2. For energies a bit above threshold and thereon, $\beta_{i} \sim 1$, or about halfway between isotropic and $\sin ^{2} \theta$. Hence it appears that the approximations made in connection with the angular distribution of photoelectrons from atomic oxygen are not valid in any spectral region, and even the assumption of constant $\beta$ is unwarranted, based upon our results.

The variation of $\beta$ with photoelectron energy $\varepsilon$ is caused principally by the variation of the phase shift difference $\xi_{d}-\xi_{s}$, with energy. This difference is made up of the sum of the Coulomb phase shift difference given by (Manson, 1973)

$$
\sigma_{d}-\sigma_{s}=-\tan ^{-1} \frac{1}{2 \sqrt{\varepsilon}}-\tan ^{-1} \frac{1}{\sqrt{\varepsilon}}
$$

and the non-Coulomb $\delta_{d}-\delta_{s}$. For oxygen at threshold $\delta_{d} \simeq \pi$, while from Equation (19) $\sigma_{d}-\sigma_{s}=-$ $\pi$, so that the phase shift difference at threshold is about $-2 \pi$. Thus the cosine term in Equation (15) is about unity, so the two terms in the numerator tend to cancel, leading to the near zero threshold value of $\beta$ shown in Figure 3. As the energy increases to $\varepsilon=1 R$, the only major change is in the Coulomb phase shift difference which becomes $\sim-\pi / 2$, so that the $\xi_{d}-\xi_{s} \simeq-3 \pi / 2$, and the cosine term in Equation (15) vanishes; $\beta$ is therefore about unity. This rapid rise of $\beta$ just above threshold is a general characteristic of atomic photoelectron angular distribution parameters and is caused by the Coulomb phase shift variations in this energy region (Kennedy and Manson, 1972b; Manson, 1973). At higher energies, no major variation in matrix elements or phase shifts occur, so $\beta$ varies only very smoothly as shown.

The adequacy of the HS approximation, as discussed above, for low- $Z$ elements gives impetus to performing further calculations using this approximation. To this end, we have calculated the angular distributions of the photoelectrons from the states arising from the ground configurations of carbon and nitrogen.

The $\beta$ 's for $\mathrm{N}$ and $\mathrm{C}$ are shown in Figure 4 . For $\mathrm{N}$, the results are substantially like $\mathrm{O}$. The $\beta_{i}$ for $\mathrm{N}$ is substantially like that for $\mathrm{O}$, since the dipole matrix elements and continuum wave phase shifts are fairly close. The variations of $\beta_{i}$ with $\varepsilon$, therefore, are results of effects discussed in connection with oxygen previously.

For carbon, the results show effects substantially the same as in $\mathrm{O}$ and $\mathrm{N}$, and for exactly the same reasons as discussed above. It is thus clear that the approximation of a constant $\beta=0$ or $\beta=2$ is quite poor in these cases as well.

\section{Final Remarks}

From the previous section it is seen that the photoelectrons from atomic oxygen have an initial angular distribution which is neither isotropic nor $\sin ^{2} \theta$. Further, we find the initial angular distribution to be strongly dependent upon the energy (wavelength) of the impinging radiation. In order to estimate the effects of this initial angular distribution on the electron density at a distant point (such as a conjugate point), it is necessary to evaluate in detail the effects of the collisions of the photoelectrons in the intervening distance as well as deflections by the Earth's magnetic field. This calculation is, however, beyond the scope of this work. In any case, we note that low energy photoelectrons will be more rapidly isotropized as shown recently by Shyn, Stolarski, and Carignan (1972). 


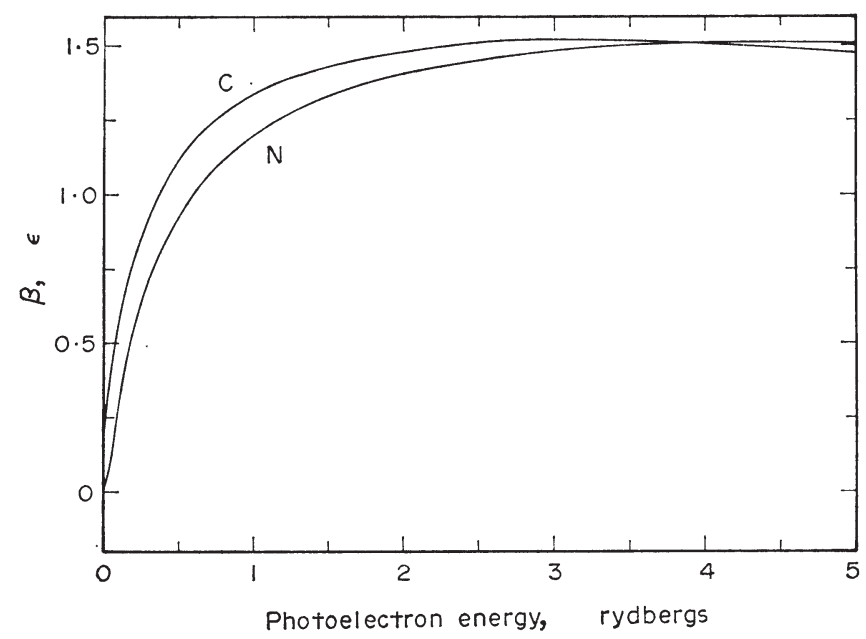

Figure 4. Asymmetry parameters, $\beta(\varepsilon)$, for $2 p$ photoionization of atomic $\mathrm{N}$ and $\mathrm{C}$ calculated using $\mathrm{HS}$ wave functions.

As to the accuracy of our calculations, comparison with experiment shows excellent agreement (Kennedy and Manson, 1972; Mitchell and Codling, 1972; Lynch, Gardner, and Codling, 1972; van der Wiel and Brion, 1973) for noble gas atoms. Some modification of the theory is necessary when fine structure is significant in the initial state of the atom, as pointed out by Dill (1973), but these effects are expected to be inconsequential here. This is presently under investigation. In addition, this work does not consider the effects of autoionization, which will affect the angular distribution in the vicinity of an inner shell threshold (Dill, 1973), in particular the $2 s$. This affects things in only a very small energy range, a few eV below the $2 s$ threshold, so it should not be too important for the conjugate point phenomenon. Finally, it has been shown theoretically by Amusia et al. (1972) that the inclusion of correlation in initial and final states significantly affects angular distributions only near Cooper minima (Cooper, 1962) and for subshells whose cross section is strongly perturbed by a nearby subshell whose cross section is very much larger via the interchannel interaction (Fano, 1961). It is thus felt that the theoretical $\beta$ 's presented herein are of sufficient accuracy for application in aeronomic problems.

\section{Acknowledgments}

Dr. Manson was supported in part by NSF Grant GP-38905, and Dr. Starace was supported in part by NASA Contract NGR 28-004-021.

\section{References}

Amusia, M., Cherepkov, N., and Chernysheva, L. (1972). Phys. Lett. 40A, 15.

Bates, D. R. (1946). Mon. Not. R. Astr. Soc. 109, 698.

Clementi, E. (1965). IBM J. Res. Dev. 9, 2 and supplement.

Cooper, J. W. (1962). Phys. Rev. 128, 681.

Cooper, J., and Zare, R. N. (1968). J. Chem. Phys. 48, 942. 
Cooper, J., and Zare, R. N. (1969). Lectures in Theoretical Physics, Vol. 11C, Atomic Collision Processes

(Eds. S. Geltman, K. Mahanthappa, and W. Brittin) p. 317. Gordon \& Breach, New York.

Dalgarno A. (1967). Rev. Mod. Phys. 39, 858.

Dalgarno, A., Henry, R. J. W., and Stewart, A. L. (1964). Planet. Space Sci. 12, 235.

Dill, D. (1973). Phys. Rev. A 7, 1976.

Dill, D., and Fano, U. (1972). Phys. Rev. Lett. 29, 1203.

Dill, D., Manson, S. T., and Starace, A. F. (1974). Phys. Rev. Lett. 32, 971, and Phys. Rev. A-to be published. Fano, U. (1961) Phys. Rev. 124, 1866.

Fano, U., and Dill, D. (1972). Phys. Rev. A 6,185.

Fontheim, E. G., Beutler, A. E., and Nagy, A. F. (1968). Annls Geophys. 24, 489.

Herman, F., and Skillman, S. (1963). Atomic Structure Calculations. Prentice-Hall, Enelewood Cliffs N.J.

Kennedy, D. J., and Manson, S. T. (1972a). Planet. Space Sci. 29, 621.

Kennedy, D. J., and Manson, S. T. (1972b). Phys. Rev. A 5, 227.

Kwei, M. W., and Nisbit, J. S. (1968). Radio Sci. 3, 674.

Lynch, M. J., Gardner, A. B., and Codung, K. (1972). Phys. Lett. 40A, 349.

Manson, S. T. (1973). J. Electron. Spectrosc. 1, 413; 2, 207.

Manson. S. T., and Cooper, J. W. (1968). Phys. Rev. 165, 126.

Manson, S. T., and Kennedy, D. J. (1970). Chem. Phys. Lett. 7, 387.

Mariani, F. (1964). J. Geophys. Res. 69, 556.

Mitchell, P., and Codung, K. (1972). Phys. Lett. 38A, 31.

Shyn, T. W., Stolarski, R. S., and Carignan, G. R. (1972). Phys. Rev. A 6, 1002.

Takayanagi, K., and Itikawa, Y. (1970). Space Sci. Rev. 11, 380.

Van der Wiel, M. J., and Brion, C. E. (1973). J. Electron. Spectrosc. 1, 439.

Whitten, R. C. (1968). J. Atmos. Terr. Phys. 30,1523.

Yang, C. N. (1948). Phys. Rev. 74, 764. 